\title{
Optimization of Cutting Interference Machining for Aircraft Roller Bearing Formation
}

\author{
Zhang Bin ${ }^{1, a}$, Cheng We $\mathrm{W}^{1, \mathrm{~b}}$ \\ ${ }^{1}$ Huali College Guangdong University Of Technology, Guangzhou Zengcheng,511325,P.R.China \\ a892962898@qq.com, b115416107@qq.com
}

Key words: aircraft; roller bearing; processing; interference; surface mesh

\begin{abstract}
In the complex components of aircraft roller bearing forming process, it is influenced by the surface mesh inducing factors, resulting in processing accuracy is not good, the error is large. An optimal roller bearing forming machining cutting method is proposed based on the internal tooth interference interpolation correction. The aircraft roller bearing forming machining constraint parameter model building and control analysis of the objective function are taken, Four single points is selected randomly for forming a curvature correction processing measurements in bearing arc at any point in solving the contact line, Newton vector intersection method is used to get the aircraft bearing roll forming and processing conditions of the contact type, spatial spectrum estimation method is used for the coherent processing, an inner tooth interference interpolation correction method taken for vehicle roller bearing machining optimization. The simulation results show that the new method has higher cutting precision, the error converges to the minimum, and the computation cost is less, and it shows a good application value.
\end{abstract}

\section{Introduction}

With the continuous development of NC machining technology, the quality of machining is constantly improving, the plates process of machining and the complexity are increased, and numerical control machining technology is widely used in the mechanical parts design and processing in the field. Roller bearing of aircraft is an important part for the flight control and power transmission of plane. By the optimal forming processing of aircraft roller bearing, it can effectively improve the flat and the flight stability and power transmission performance. Therefore, the study of aircraft roller bearing forming optimization processing technology has a wide range of application value in the field of aircraft manufacturing, and the related mechanical processing technology research has been obtained great attention of people.

Types of mechanical processing are more, such as welding, cutting and grinding process, in the vehicle roller bearing forming processing, it needs for cutting processing, through cutting interference processing, and the plates are processed assembly for subsequent grinding plate. Traditionally, aircraft roller bearing cutting interference processing method is taken based on internal tooth interference, and the contact line along the tooth width curvature correction of vehicle roller bearing cutting interference processing method, and processing parameter adaptive weighted vehicle roller bearing cutting interference processing method[1-3]. With the in-depth study of digital processing control technology and the development of processing modification optimization algorithm, the research in vehicle roller bearing cutting interference processing area has made some achievements. Among them, the reference [4], it proposed a 2-axis NC machining and fuzzy neural network control method for vehicle roller bearing cutting, to realize the machining error compensation and fuzzy control, but the method of $\mathrm{NC}$ machining prone to thermal error. In reference [5], it proposed a vehicle roller bearing cutting machining interference control model based on contour error compensation of linear track, to improve the processing performance, but this method easily has feeding error, effects on the roller bearing machining accuracy. In view of the above problems, this paper proposes an optimal vehicle roller bearing forming machining method based on the internal tooth interference interpolation correction, the vehicle roller bearing forming machining constraint parameter model is constructed and control analysis of the objective function is obtained, 
and then an inner tooth interference interpolation correction method is used for vehicle roller bearing machining optimization, simulation experiments are taken, it shows the superiority of this method.

\section{Basic knowledge and constraint parameters analysis of forming process of aircraft roller bearing}

\subsection{Tooth model and coordinate construction of aircraft roller bearings machining}

In order to realize the roller bearing cutting processing of machining, first, the analysis of tooth model in cutter spiral guide process under the interference is taken, if aircraft roller bearing forming machining process of the left tooth face gradually array is composed of $M$ transition surfaces for pitting and dedendum line array, the tooth model of flight for roller bearing forming processing is shown in Figure 1.

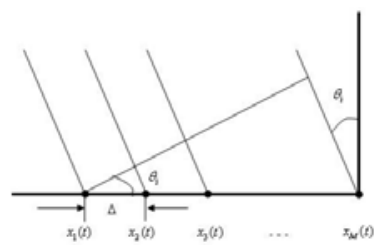

Figure 1. Tooth model of aircraft roller bearing forming

In the figure, the transition curve is distributed in an arbitrary spherical mesh, and the root stress should be randomly distributed in space plan, involute meshing starting point has $d$ profiles of grinding wheel, it is shown by $s_{i}(t)(i=1,2, \ldots, d)$, parameters such as radius and position is decided by the $\mathrm{M}$ array outputs, and the control objective function is expressed as:

$$
x_{m}(t)=\sum_{i=1}^{d} g_{m}\left(\theta_{i}\right) s_{i}\left[t-\tau_{m}\left(\theta_{i}\right)\right]+n_{m} \quad(m=1,2, \mathrm{~L}, M)
$$

In the formula, $\theta_{i}$ is the the subsequent flow acceleration pushing direction of the $\mathrm{i}$-th transition curve, $g_{m}\left(\theta_{i}\right)$ is $3 \mathrm{D}$ rail detection sensitivity of high resolution micro spray gun for machining in base array, $\tau_{m}\left(\theta_{i}\right)$ is forming size in time domain for spectrum estimation, the i-th cutter cutting speed is obtained. $n_{m}$ is single point forming and single forming interference vector of ${ }^{m}$-th the array. It is an array, the output of single point forming and single line forming can be described as a dimension vector, which is expressed by a matrix:

$$
\left[\begin{array}{c}
x_{1}(t) \\
x_{2}(t) \\
\mathrm{M} \\
x_{M}(t)
\end{array}\right]=\left[\begin{array}{c}
\sum_{i=1}^{d} g_{1}\left(\theta_{i}\right) s_{i}\left[t-\tau_{1}\left(\theta_{i}\right)\right] \\
\sum_{i=1}^{d} g_{2}\left(\theta_{i}\right) s_{i}\left[t-\tau_{2}\left(\theta_{i}\right)\right] \\
\mathrm{M} \\
\sum_{i=1}^{d} g_{M}\left(\theta_{i}\right) s_{i}\left[t-\tau_{M}\left(\theta_{i}\right)\right]
\end{array}\right]+\left[\begin{array}{c}
n_{1}(t) \\
n_{2}(t) \\
\mathrm{M} \\
n_{M}(t)
\end{array}\right]
$$

The single point forming sample in the cutting feed direction vector can be described in space frequently domain independently of each other, then for the single shaping sample, the optimization of processing feed control equation can be compacted as:

$$
X(t)=G S(t)+n(t)
$$

Wherein

$$
\begin{gathered}
X(t)=\left[\begin{array}{llll}
x_{1}(t) & x_{2}(t) & \mathrm{L} & \left.x_{M}(t)\right]^{T} \\
G=\left[\begin{array}{cccc}
g_{1}\left(\theta_{1}\right) & g_{1}\left(\theta_{2}\right) & \mathrm{M} & g_{1}\left(\theta_{d}\right) \\
g_{2}\left(\theta_{1}\right) & g_{2}\left(\theta_{2}\right) & \mathrm{M} & g_{2}\left(\theta_{d}\right) \\
\mathrm{L} & \mathrm{L} & \mathrm{O} & \mathrm{L} \\
g_{M}\left(\theta_{1}\right) & g_{M}\left(\theta_{2}\right) & \mathrm{M} & g_{M}\left(\theta_{d}\right)
\end{array}\right] \\
S_{i}(t)=\left[s_{i}\left(t-\tau_{1}\left(\theta_{i}\right)\right)\right. & s_{i}\left(t-\tau_{2}\left(\theta_{i}\right)\right) & \mathrm{L} & \left.s_{i}\left(t-\tau_{M}\left(\theta_{i}\right)\right)\right]
\end{array}\right.
\end{gathered}
$$


According to the above analysis, the coordinate system model of the forming machining of the roller bearing is expressed as:

$$
v(t, \theta)=\sum_{m=1}^{M} \omega_{i}^{*}(\theta) x_{i}(t)=\sum_{m=1}^{M} x_{i}^{*}(t) \omega_{i}(\theta)
$$

In the formula, "*" represents the complex conjugate operator, in the above coordinate system, the constraint parameters model is constructed to optimize the machining process, and the control objective function is analyzed to improve the precision of the interference process.

\subsection{Analysis and optimization of processing parameter constraint model}

According to the above construction of the tooth model and coordinate system of vehicle roller bearing machining, using the modular modeling method, assuming the vehicle forming cutting roller bearing has eight solid elements, forming cutting roller bearings based on the interference process of heat conduction principle[6-10], the output shaft formed the department of mechanical components of roller bearing forming cutting interference process. With the change of roller bearing forming cutting interference point heat dissipation coefficient of fatigue damage, get the i-th unit of the M array workpiece of interference points on line width for forming the output gain:

$$
\tau_{m}\left(\theta_{i}\right)=(m-1) \tau_{0}\left(\theta_{i}\right)=(m-1) \frac{\Delta}{c} \sin \theta_{i} \quad(m=1,2, \mathrm{~L}, M)
$$

Where, $\tau_{0}\left(\theta_{i}\right)=\frac{\Delta}{c} \sin \theta_{i}$ is the single point forming of linear size controllable time delay, $\mathrm{c}$ is the heat transfer coefficient of the two vertical directions in the medium, then through layer scanning, the constraint parameter model by cutting interference is:

$$
\left[\begin{array}{c}
x_{1}(t) \\
x_{2}(t) \\
\mathrm{M} \\
x_{M}(t)
\end{array}\right]=\left[\begin{array}{c}
\sum_{i=1}^{d} g_{1}\left(\theta_{i}\right) s_{i}(t) \\
\sum_{i=1}^{d} g_{2}\left(\theta_{i}\right) s_{i}\left(t-\frac{\Delta}{c} \sin \theta_{i}\right) \\
\mathrm{M} \\
\sum_{i=1}^{d} g_{M}\left(\theta_{i}\right) s_{i}\left(t-(M-1) \frac{\Delta}{c} \sin \theta_{i}\right)
\end{array}\right]+\left[\begin{array}{c}
n_{1}(t) \\
n_{2}(t) \\
\mathrm{M} \\
n_{M}(t)
\end{array}\right]
$$

Among them, according to the characteristics of the 16 interference information processing entities control area channel sampling, with characteristic value drop value arranged by spraying pressure cutting, optimization control technology is proposed, using the plane filtering method, spatial gain is focused, it is:

$$
\left[\begin{array}{c}
x_{1}(t) \\
x_{2}(t) \\
\mathrm{M} \\
x_{M}(t)
\end{array}\right]=\left[\begin{array}{c}
\sum_{i=1}^{d} s_{i}(t) \\
\sum_{i=1}^{d} s_{i}\left(t-\frac{\Delta}{c} \sin \theta_{i}\right) \\
\mathrm{M} \\
\sum_{i=1}^{d} s_{i}\left(t-(M-1) \frac{\Delta}{c} \sin \theta_{i}\right)
\end{array}\right]+\left[\begin{array}{c}
n_{1}(t) \\
n_{2}(t) \\
\mathrm{M} \\
n_{M}(t)
\end{array}\right]
$$

On the basis of this, the optimization of processing control is taken.

\section{Optimization of roller bearings cutting interference machining}

According to the above construction processing constraint parameter model and coordinate system, cutting interference processing optimization design of forming roller bearings, the traditional method used curvature error correction method with stratified cutting, machining complexity increases the contact line in the coordinate system of the grinding wheel interference error caused by surface, machining accuracy is not good, the efficiency and accuracy of processing is limited, $f$, in order to overcome the drawbacks of traditional methods. An optimal roller bearing forming machining cutting method is proposed based on the internal tooth interference interpolation correction. The aircraft roller bearing forming machining constraint parameter model building and control analysis of the 
objective function are taken. The fatigue load radial roller bearing clearance forming cutting interference process meet the single fluid mass conservation, it is:

$$
\frac{\partial \rho}{\partial t}+\frac{\partial}{\partial x_{i}}\left(\rho u_{i}\right)=S_{m}
$$

The momentum equation of roller bearing forming cutting interference under the center of curvature of path planning is:

In the formula, wherein:

$$
\frac{\partial}{\partial t}\left(\rho u_{i}\right)+\frac{\partial}{\partial x_{j}}\left(\rho u_{i} u_{j}\right)=-\frac{\partial p}{\partial x_{i}}+\frac{\partial \tau_{i j}}{\partial x_{j}}+\rho g_{i}+F_{i}
$$

$$
\tau_{i j}=\left[\mu\left(\frac{\partial u_{i}}{\partial x_{j}}+\frac{\partial u_{j}}{\partial x_{i}}\right)\right]-\frac{2}{3} \mu \frac{\partial u_{i}}{\partial x_{i}} \delta_{i j}
$$

Each roller bearing forming cutting interference trajectory point is determined in time, and Newton iterative equations satisfy the control point of contact with the grinding wheel surface of the spiral surface:

$$
\frac{\partial \rho}{\partial t}=0, \frac{\partial}{\partial t}\left(\rho u_{i}\right)=0
$$

Assuming the grinding of high precision small error does not exist under the disturbance source, therefore $S_{m}=0$, assuming there is no the external pressure, by using numerical simulation, obtained $F_{i}=0$. Ignore the effect of the axial cross-section of the wheel with gravity, then: $\rho g_{i}=0$.

The polynomial interpolation method is used for constructing turbulence equation $k-\varepsilon$, which is expressed as:

$$
\begin{array}{r}
\frac{\partial}{\partial t}(\rho k)+\frac{\partial}{\partial x_{i}}\left(\rho k u_{i}\right)=\frac{\partial}{\partial x_{j}}\left[\left(\mu+\frac{\mu_{t}}{\sigma_{k}}\right) \frac{\partial k}{\partial x_{j}}\right]+G_{k}+G_{b}-\rho \varepsilon-Y_{M}+S_{k} \\
\frac{\partial}{\partial t}(\rho \varepsilon)+\frac{\partial}{\partial x_{i}}\left(\rho \varepsilon u_{i}\right)=\frac{\partial}{\partial x_{j}}\left[\left(\mu+\frac{\mu_{t}}{\sigma_{\varepsilon}}\right) \frac{\partial \varepsilon}{\partial x_{j}}\right]+C_{1 \varepsilon}\left(G_{k}+C_{3 \varepsilon} G_{b}\right)-C_{2 \varepsilon} \rho \frac{\varepsilon^{2}}{k}+S_{\varepsilon}
\end{array}
$$

Where, the contact line of involute part meets:

$$
\mu_{t}=\rho C_{\mu} \frac{k^{2}}{\varepsilon}
$$

According to the above parameters, and roller bearing tooth surface equations is combined, in grinding wheel coordinates, equilibrium control equation is obtained as:

$$
\phi=1-\sqrt{\left(x-x_{0}\right)^{2} / r_{0}^{2}+\left(y-y_{0}\right)^{2} / r_{0}^{2}}
$$

Where, ${ }^{x_{0}}$ and $y_{0}$ are the gear involute spiral surface normal vector and the processing center, the error fitting error correction test method of interference spectrum is taken, this process is described with Lyapunove equation:

$$
C=\operatorname{Min}\left\{\max \left(C_{i}\right)\right\}, \sum_{j^{\prime}=1}^{p^{\prime}} Z_{i, j, j^{\prime}}=1, \quad \forall i \in(1, n), \quad \forall j \in\left(1, n_{i}\right)
$$

Where, $C_{i}$ is the confidence of any point of the roller bearing surface of revolution, $Z_{i, j, j}$ is the discrete sample spectrum composed of sand profile of a plurality of contact points, the extreme value problem is transferred into seeking the following objective function for processing feed:

$$
\mu_{i k}=1 / \sum_{j=1}^{c}\left(d_{i k} / d_{j k}\right)^{\frac{2}{m-1}}, \quad V_{i}=\sum_{k=1}^{m}\left(\mu_{i k}\right)^{m} x_{k} / \sum_{k=1}^{n}\left(\mu_{i k}\right)^{m}
$$

The chaotic differential disturbance is taken for the curved forming error correction in the cutting process, using the direct method to obtain curvature calculation formula:

$$
A=U S V^{\prime}=U\left(\begin{array}{rr}
\sum & 0 \\
0 & 0
\end{array}\right) V^{\prime}, U * U^{\prime}=I, V * V^{\prime}=I
$$


Where, $\mathrm{A}$ is $m \times n$ dimensional matrix, $A * A^{\prime}$ and $A^{\prime} * A$ are main component features. And through the above treatment, combined with internal tooth interference interpolation, cutting vehicle roller bearing forming interference processing optimization is realize.

\section{Simulation experiments and results analysis}

In order to test the performance of the correction and optimization in aircraft cutting roller bearing forming interference processing and aircraft roller bearing machining, the computer simulation is taken. The experiment is constructed based on the structure model of CAD/CAM system, the hardware environment if the frequency of $2.8 \mathrm{GHz}, 2 \mathrm{G}$ RAM, windows XP operating system, the software design uses $\mathrm{CAD}$ for $\mathrm{CNC}$ machining, according to the simulation environment and parameter setting, the tooth interference interpolation parameters respectively: $C_{1 \varepsilon}=1.44, C_{2 \varepsilon}=1.92$, the first volume of basic surface $C_{\mu}=0.09, \sigma_{k}=1.0$, helix angle $\sigma_{\varepsilon}=1.3$. The constraint parameter convergence process for machining of the aircraft roller bearing is shown in Figure 2.

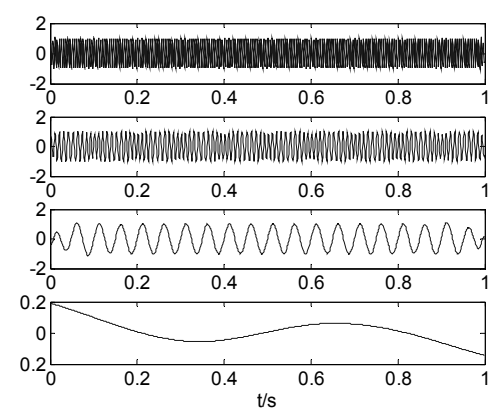

Figure 2. Constraint parameters convergence curves

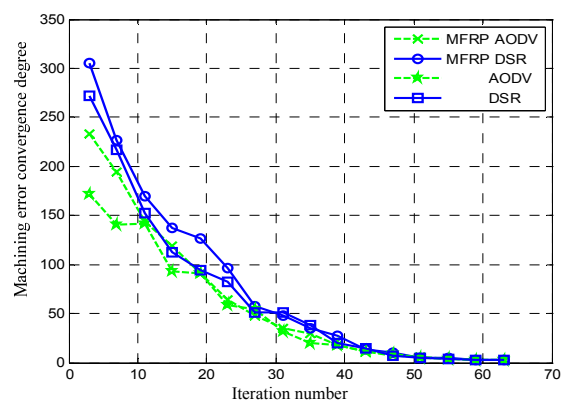

Figure 3. Comparison of performance test

It can be seen from the diagram, using the method, the constraints in the interference area is gradually widened, converge to the optimal value, thereby reducing unnecessary error correcting operation, improve the machining accuracy, in order to compare the performance of the algorithm, using this algorithm and the traditional method, the machining error is taken as the test index, get the simulation results shown in Figure 3, it can be seen in Figure 3, using this processing method, it has smaller error on aircraft roller bearing processing, and it shows the application performance of the algorithm is better.

\section{Conclusions}

Aircraft roller bearing is an important component of aircraft flight control and power transmission, through the optimization of forming roller bearing of aircraft, and it can effectively improve the flight stability and power transmission and security. This paper presents a method to optimize machining interpolation aircraft forming roller bearing interference based on inner gear, build the control objective function was obtained, and analysis of constraint parameter model of roller bearings for machining aircraft were carried out, then the internal tooth interference correction method for interpolation was used to optimize machining roller bearing of aircraft. Research result shows that 
using this method to optimize the process, it can reduce the error, and has good performance, it shows good application value.

\section{Acknowledgments}

2012 the quality project of Guangdong province "electromechanical integrated skills training cen ter" (Guangdong teach high letter [2012] No. 204) and institutions of higher learning in Guangdong province in 2014 "comprehensive reform pilot project" .

\section{References}

[1] GUO Erkuo, HUANG Xiaodiao, YUAN Hong, et al. Optimization of contact line for form grinding involute helical gears[J]. Mechanical Science and Technology for Aerospace Engineering, 2012, 31(8): 1320-1324.

[2] FAN Wengang, YE Peiqing. Research Progress in Tool Path Planning for Five-axis End Milling Machining of Sculptured Surfaces. Journal of Mechanical Engineering, 2015, 51(15): 168-182.

[3] SUN Kehui, HE Shaobo, DONG Yanqing. Tracking Synchronization Control of the Simplified Lorenz Chaotic System[J]. Information and control, 2015,44(4): 393-397.

[4] Min F H, Wang Z Q. Generalized projective synchronization and tracking control of complex Dynamos chaotic systems[J]. Acta Physica Sinica, 2008, 57(1): 31-36.

[5] REN Zhaohui, XIE Jixiang, ZHOU Shihua, WEN Bangchun. Vibration Characteristic Analysis of Helical Gear-rotor-bearing System with Coupled Lateral-torsional-axial. Journal of Mechanical Engineering, 2015, 51(15): 75-89.

[6] ZHANG Wenjia, SHANG Weiwei. High-speed Point-to-point Trajectory Planning of a 2-DOF Cable Driven Parallel Manipulator. Journal of Mechanical Engineering, 2016, 52(3): 1-8.

[7] GOSSELIN C, FOUCAULT S. Dynamic point-to-point trajectory planning of a two-DOF cable-suspended parallel robot[J]. IEEE Transactions on Robotics, 2012: 1476-1481.

[8] BARRETTE G, GOSSELIN C. Determination of the dynamic workspace of cable-driven planar parallel mechanisms[J]. ASME J. Mech. Design, 2005， 127(2): 242-248.

[9] DING Guolong, ZHANG Song, ZHAO Daxing, ZHAO Dongxiong. Interference Study of Gear Form Grinding Based on Induced Normal Curvature. Journal of Mechanical Engineering, 2016, 52(3): 197-204.

[10] CHEN Xinchun, LI Jia, LOU Benchao, et al. Effect of the cutter parameters and machining parameters on the interference in gear slicing $[\mathrm{J}]$. Chinese Journal of Mechanical Engineering, 2013, 26(6): 1118- 1126. 